\title{
Substrate Properties of Fluorescent Ribonucleo- tides in the Terminal Transferase-Catalyzed Labeling of DNA Sequencing Primers
}

BioTechniques 21:1084-1092 (December 1996)

\author{
Gabor L. Igloi \\ Institut für Biologie III, \\ Universität Freiburg, Freiburg, \\ Germany
}

\begin{abstract}
Terminal deoxynucleotidyltransferase (terminal transferase, E.C. 2.7.7.31) has been used to add a single fluorescent ribonucleotide to the $3^{\prime}$ terminus of DNA sequencing primers, thereby creating primers suitable for automated DNA sequence analysis. The previously introduced procedure using fluorescein-UTP for the postsynthetic labeling of primers can, under appropriate reaction conditions, now be extended to commercially available fluorescein-ATP and fluorescein-CTP permitting greater flexibility in primer design. The products of these addition reactions have been shown to provide sequence data qualitatively and quantitatively identical to those obtained with conventional $5^{\prime}$-terminally labeled primers using cycle sequencing conditions in conjunction with an automated sequencer. Ribonucleotide derivatives of four other dyes (coumarin, tetramethylrhodamine, lissamine and Texas Red $\left.{ }^{\circledR}\right)$ were also examined for their potential in the terminal transferase-catalyzed reaction. Whereas coumarin-UTP was efficiently incorporated giving a monoaddition product, the conjugates of all other dyes with ATP, CTP and UTP were extremely poor substrates under all conditions tested.
\end{abstract}

\section{INTRODUCTION}

Terminal deoxynucleotidyl transferase is one of several enzymes whose importance in molecular biology has not been in its function in vivo, but rather as a tool for manipulating the $3^{\prime}$ termini of DNA. Its value lies, atypically for an enzyme, in its flexible specificity, a property that was recognized at a time when it was under initial enzymatic scrutiny (9). The addition of ribonucleotides (5) to DNA, of importance in early DNA sequencing (14), has once again proven to be valuable in the development of automated DNA sequencing strategies. It was previously shown (4) that terminal transferase will add a single fluorescein-riboUTP to the $3^{\prime}$ terminus of a sequencing primer. Modification at this position does not influence the elongation of the primer, either during DNA polymerase-catalyzed sequencing or during polymerase chain reaction (PCR). A limitation of this enzymatically unique procedure of attaching a fluorescent label to a pre-formed sequencing primer, lay in the restricted commercial availability of labeled nucleotides. Recently, however, many more variously labeled ribonucleotides have appeared on the market so that an extension of the previously described labeling reaction, both in regards to other nucleotides as well as to other dyes, has become feasible. This report describes the substrate properties of 14 commercial fluorescent ribonucleotides. Emphasis is placed on the extension of our previous protocol (3) to fluorescein-riboCTP and fluorescein-riboATP (fluoresceinriboGTP is, as yet, not available) and the use of primers labeled in this way during automated DNA sequencing.

\section{MATERIALS AND METHODS}

Oligonucleotides were synthesized by standard phosphoramidite methods using an Applied Biosystems 394 system (Perkin-Elmer/Applied Biosystems Division [PE/ABI], Foster City, CA, USA). Following cleavage and deprotection (OligoPrep; Savant, Farmingdale, NY, USA), they were subjected to one round of ethanol precipitation with $0.3 \mathrm{M} \mathrm{NaOAc}, \mathrm{pH}$ 4.8. Unless otherwise stated, for the systematic study of labeling efficiency, the universal M13 primer (5' GTAAAACGACGGCCAGTG $3^{\prime}$ ) was used. Primers bearing a fluorescent label at the $5^{\prime}$ terminus were synthesized using FluorePrime ${ }^{\circledR}$ fluorescein amidite (Pharmacia Biotech, Uppsala, Sweden). Terminal transferase was from Boehringer Mannheim (Mannheim, Germany) or MBI Fermentas (Vilnius, Lithuania) (see also Reference 13), and the reaction buffer was supplied with the enzyme unless otherwise indicated. Fluorescein-allylamino-UTP was the product of Boehringer Mannheim; all other labeled nucleotides were obtained from NEN Life Sciences (Bad Homburg, Germany) (Figure 1). The spectroscopic properties of the dyes are summarized in Table 1. Labeling reactions were carried out as described previously $(3,4)$ using the incubation conditions indicated in the figure legends or in Table 2. For the labeling of primers destined to be used in DNA sequencing, a more streamlined protocol may be used. The incubation then contains $0.2 \mu \mathrm{g}$ primer, $10 \mu \mathrm{M}$ fluorescein-rNTP, $2 \mu \mathrm{L} 5 \times \mathrm{TdT}$ buffer, $0.75 \mathrm{mM} \mathrm{CoCl}_{2}$ and $15 \mathrm{U}$ terminal transferase in a total volume of 10 $\mu \mathrm{L}$. After $30 \mathrm{~min}$ at $37^{\circ} \mathrm{C}$, the enzyme is inactivated at $95^{\circ} \mathrm{C}$ for $2 \mathrm{~min}$ and 1 
Table 1. Optical Properties of the Fluorophores Attached to the Nucleotide Substrates

\begin{tabular}{|lcc|}
\hline Fluorophore & Excitation $\mathbf{( n m})(\mathbf{p H ~ 7 . 0 )}$ & Emission (nm) $\mathbf{( p H ~ 7 . 0 )}$ \\
\hline Coumarin & 402 & 443 \\
Fluorescein & 494 & 517 \\
Tetramethylrhodamine & 550 & 570 \\
Lissamine & 570 & 588 \\
Texas Red & 593 & 612 \\
\hline
\end{tabular}

$\mu \mathrm{L}$ of the solution may then be used directly, without further purification, in the sequencing reaction. Incorporation of a fluorescent label was monitored by gel electrophoresis of the products on a $20 \% 7 \mathrm{M}$ urea polyacrylamide gel. The fluorescent bands were detected by trans-illumination at $254 \mathrm{~nm}$ and recorded using a Cybertech CS1 video system (Berlin, Germany). The negative image is depicted. Bands corre- sponding to both labeled and non-fluostaining with Stains-All (Serva, Heidelberg, Germany). For cycle sequencing of DNA, SequiTherm ${ }^{\mathrm{TM}}$ DNA polymerase (Epicentre Technologies, Madison, WI, USA) was used according to the manufacturer's instructions in a GeneAmp ${ }^{\circledR}$ PCR System 9600 (PerkinElmer, Ueberlingen, Germany) thermal cycler programmed to give 36 cycles of rescent primers were visualized by $94^{\circ} \mathrm{C}$ for $20 \mathrm{~s} ; 50^{\circ} \mathrm{C}$ for $20 \mathrm{~s}$ and $72^{\circ} \mathrm{C}$ for $40 \mathrm{~s}$, with an initial denaturing step at $94^{\circ} \mathrm{C}$ for $5 \mathrm{~min}$ and a final $72^{\circ} \mathrm{C}$ soak for $7 \mathrm{~min}$. When using Thermo Sequenase $^{\mathrm{TM}}$ (Amersham, Braunschweig, Germany), 250 ng plasmid DNA in 5 $\mu \mathrm{L}$ were added to each of four $2-\mu \mathrm{L}$ dNTP mixtures containing 7-deazadGTP plus the polymerase and $1 \mu \mathrm{L}(5$ ng) labeled primer in a thin-walled 0.2$\mathrm{mL}$ PCR tube (Perkin-Elmer). Cycling was performed for 25 cycles of $94^{\circ} \mathrm{C}$ for $30 \mathrm{~s}$ and $60^{\circ} \mathrm{C}$ for $30 \mathrm{~s}$ with an initial 1-min denaturing at $95^{\circ} \mathrm{C}$. After the addition of $5 \mu \mathrm{L}$ of $10 \mathrm{mg} / \mathrm{mL}$ Blue Dextran and $1 \mathrm{mM}$ EDTA in formamide and denaturing at $95^{\circ} \mathrm{C}$ for 3 $\min , 4.5 \mu \mathrm{L}$ were applied to a $6 \%$ sequencing gel in an A.L.F. ${ }^{\text {TM }}$ DNA Sequencer (Pharmacia Biotech, Piscataway, NJ, USA). It should be

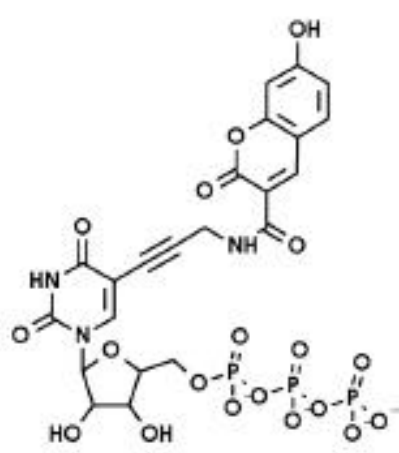

Coumarin alkynylamino-UTP

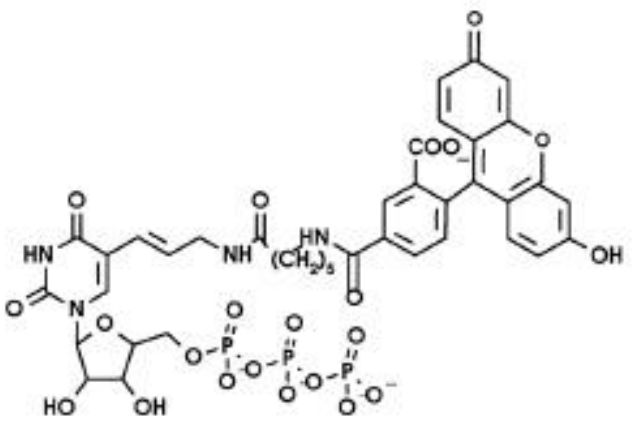

Fluorescein allylamino-UTP

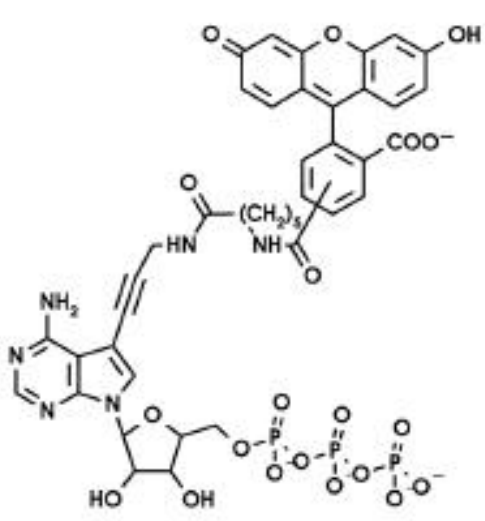

Fluorescein alkynylamino-ATP

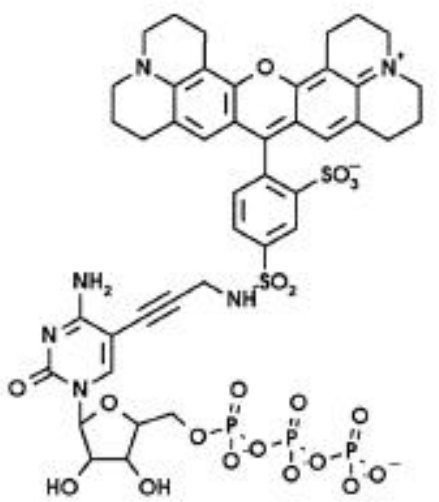

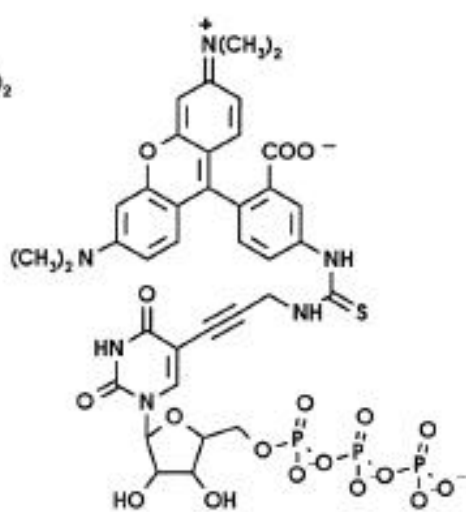

Tetramethylrhodamine alkynylamino-UTP

Texas Red alkynylamino-CTP

Figure 1. Structure of selected representative nucleotide-dye derivatives used in this study. 
emphasized that for primers labeled using this protocol, $\mathrm{NaOH}$ should NOT be present in the denaturing buffer since this leads to the hydrolysis of the ribo-linkage between the primer and the first base added by the DNA polymerase.

\section{RESULTS}

\section{Substrate Properties of Fluorescent NTPs}

Initial screening of the labeled NTPs in the terminal transferase-catalyzed reaction indicated that the reaction conditions required optimization to give the desired mono-addition product; in some cases multiple additions were observed (fluorescein-ATP), in others the labeling efficiency was minimal (Texas Red $^{\circledR}$-CTP) (see below). A set of 9 reaction conditions for each nucleotide was selected and each nucleotide was subjected to those conditions.

A. Fluorescein-alkynylamino-ATP, -CTP and -UTP, and fluoresceinallylamino-UTP. The set of fluorescein-labeled NTPs in which the label was attached to the base by means of an acetylinic link (alkynylamino-NTPs) were good substrates for terminal transferase. However, the yield of addition product depended on the nature of the nucleotide. Under conditions where efficient $(>60 \%)$ mono-addition of fluorescein-UTP took place, there was a significant yield of dinucleotide addition for fluorescein-ATP. This di-addition (or even tri-addition) (Figure 2A) was maintained under all conditions where significant activity was observed. Multiple additions of labeled nucleotides to sequencing primers has been shown to reduce the signal intensity generated by the sequencing reaction (4). A closer examination of the reaction products showed, however, that the undesired multiple addition was reduced somewhat in the presence of dimethyl sulfoxide (DMSO) (lane 4) and in phosphate buffer (lane 6), while still supporting efficient labeling. A combination of these parameters had the desired additive effect of virtually eliminating multiple additions (Figure 2B, lane 1). Nevertheless, even under these conditions, di-addition (to an extent of $24 \%$ of the mono-adduct, in the case of fluorescein-ATP) could be induced by increasing the fluorescent-NTP concentration from the routine $0.1 \mathrm{mM}$ (lane 2) to $0.4 \mathrm{mM}$ (lane 4). A direct comparison of the labeling efficiency of allylamino-linked fluorescein-UTP with the alkynylamino derivative of UTP under optimal incorporation conditions (Figure 3) showed no difference between these two species, which only differed by the state of the unsaturated C-C link and its associated conformational constraints (Figure 1). The labeling efficiency using the alkynyl-NTPs under three sets of buffer conditions is shown in Figure 4, while their quantification is summarized in Table 3.

B. Tetramethylrhodamine-alkynylamino-UTP, -alkynylamino-CTP and -alkynylamino-ATP. The tetramethylrhodamine derivatives of ATP, CTP and UTP were investigated. Optimal activity was attained in cacodylate/DMSO, but even then an incorporation of only $24 \%$ was observed with the UTP derivative. The corresponding ATP and CTP species were poorer

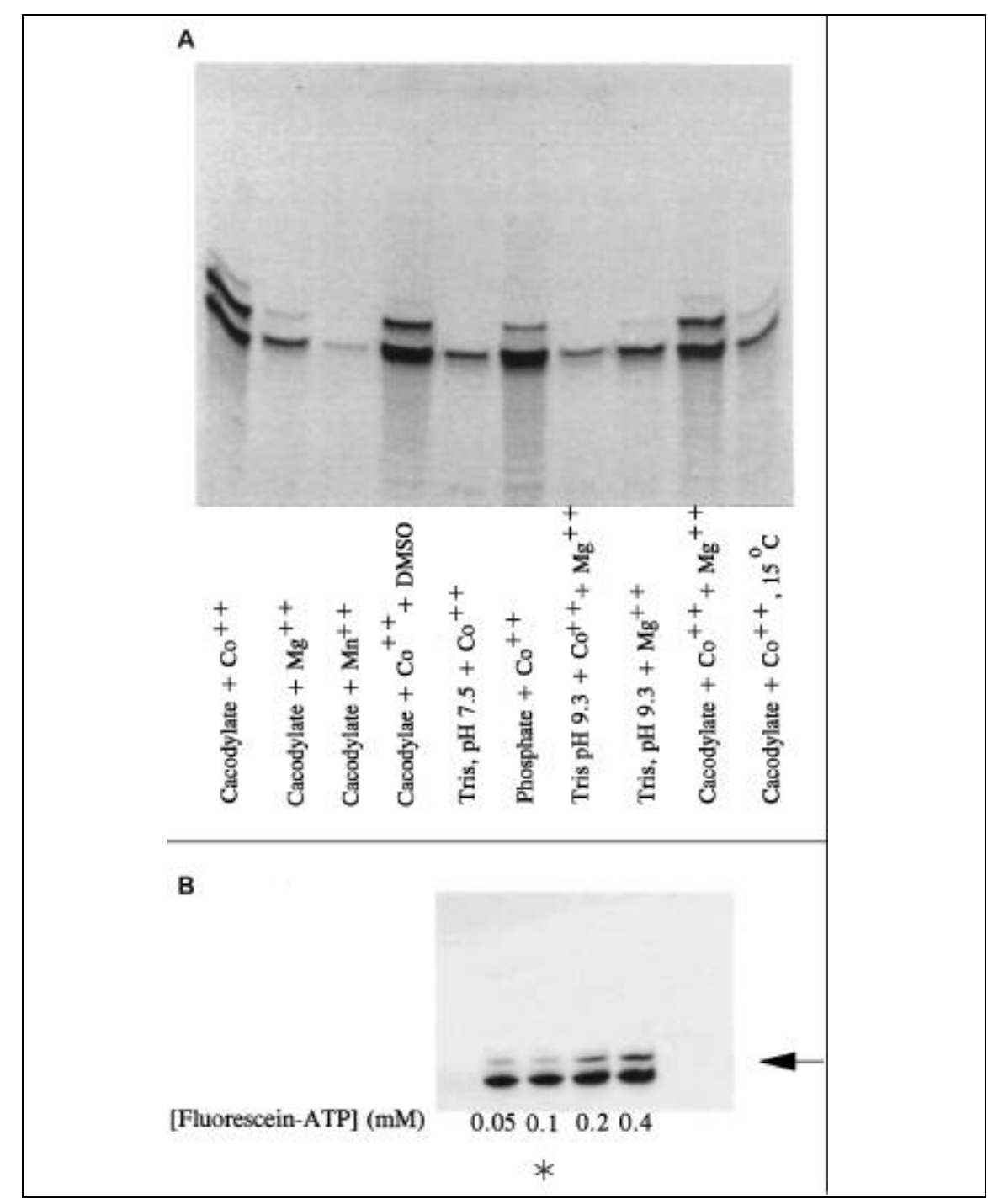

Figure 2. Addition of fluorescein alkynylamino-ATP to the $3^{\prime}$ terminus of an oligonucleotide. The reactions were carried out as described in Materials and Methods and as detailed in Table 2. The electrophoretic analysis depicts the electronic conversion of the intrinsic fluorescent image to its negative form and cannot be compared quantitatively with the results obtained using Stains-All in Figures 3 and 4. Panel A: Reaction products obtained using each of the nine reaction buffers in Table 2, as indicated, together with a reaction in cacodylate $+\mathrm{Co}^{++}$at $15^{\circ} \mathrm{C}$. Panel B: Nucleotide concentration dependence of fluorescein alkynylamino-ATP incorporation in phosphate buffer (Reaction No. 6, Table 2) plus 10\% DMSO. The concentration of nucleotide was varied as indicated. An arrow marks the position of diaddition product; an asterisk, the standard nucleotide concentration. 
substrates (Table 3).

C. Lissamine-alkynylamino-ATP, -CTP and -UTP. The lissamine-coupled nucleotides, whose dye residue is structurally closely related to tetramethylrhodamine (Figure 1), yet with spectroscopic properties (Table 1) that approach those of Texas Red, proved to be a poorer substrate than the former and showed barely detectable incorporation (Table 3), even under optimal conditions.

D. Texas Red-alkynylamino-ATP, -CTP and -UTP. Analysis of Texas Red-labeled NTPs showed that these nucleotides were extremely poor substrates in this reaction. Screening using the nine selected reaction conditions only gave weak signals with reactions 1,4 and 9 (data not shown). All attempts at improving the labeling efficiency by using common additives such as spermidine, polyethylene glycol or hexammine cobalt chloride, prolonging the incubation time or using more

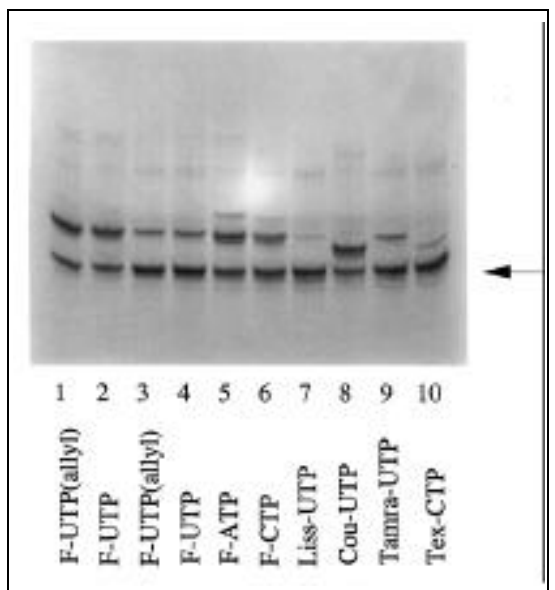

Figure 3. Comparison of the incorporation of fluorescent nucleotides under conditions determined as being optimal for each species indicated. Reactions were carried out as described in Materials and Methods. The bands were located by staining with Stains-All and quantified by densitometry (Table 3). Band visible just below the main mono-addition products are addition products derived from the primer n-1 species, also seen below the band of the unlabeled species. Abbreviations: F, fluorescein; Liss, lissamine; Cou, coumarin; Tamra, tetramethylrhodamine; Tex, Texas Red. Unless otherwise stated, the dyes are attached to the nucleotides via alkynylamino links. Buffer conditions were cacodylate + DMSO (reaction 4, Table 2), lanes 1 , 2, 7-10; and phosphate + DMSO (reaction $6+$ DMSO, Table 2), lanes 3-6. Note the anomalous migration of the primer labeled with Texas RedCTP. The position of the unlabeled primer is marked by an arrow.

Table 2. Reaction Conditions Used for Screening the Substrate Properties of Fluorescent Nucleotides

\begin{tabular}{|c|c|c|c|c|c|c|c|c|c|}
\hline \multirow[b]{2}{*}{ Components } & \multicolumn{9}{|c|}{ Reaction Conditions } \\
\hline & 1 & 2 & 3 & 4 & 5 & 6 & 7 & 8 & 9 \\
\hline $\begin{array}{c}200 \text { mM K-cacodylate, } \\
25 \text { mM Tris, pH } 6.6\end{array}$ & 2 & 2 & 2 & 2 & - & - & - & - & 2 \\
\hline $5 \mathrm{mM} \mathrm{CoCl}_{2}$ & 1.5 & - & - & 1.5 & 1.5 & 1.5 & 1.5 & - & 1.5 \\
\hline $100 \mathrm{mM} \mathrm{MgCl}_{2}$ & - & 0.5 & - & - & - & - & - & - & 0.5 \\
\hline $100 \mathrm{mM} \mathrm{MnCl}_{2}$ & - & - & 0.5 & - & - & - & - & - & - \\
\hline $1 \mathrm{M}$ Tris-HCl, $\mathrm{pH} 7.5$ & - & - & - & - & 0.5 & - & - & - & - \\
\hline DMSO & - & - & - & 1 & - & - & - & - & - \\
\hline $1 \mathrm{M} \mathrm{K}$-phosphate, $\mathrm{pH} 7.2$ & - & - & - & - & - & 0.5 & - & - & - \\
\hline $\begin{array}{l}500 \text { mM Tris- } \mathrm{HCl}, \mathrm{pH} \text { 9.3, } \\
25 \mathrm{mM} \mathrm{MgCl}_{2}\end{array}$ & - & - & - & - & - & - & 1 & 1 & - \\
\hline \multicolumn{10}{|c|}{$\begin{array}{l}\text { The final reaction volume was } 10 \mu \mathrm{L} \text {, including } 3 \mu \mathrm{g} \text { primer, } 0.1 \mathrm{mM} \text { fluorescent } \\
\text { nucleotide and } 15 \mathrm{U} \text { terminal transferase. All numbers refer to volumes in } \mu \mathrm{L} \text {. The } \\
\text { incubation temperature was } 37^{\circ} \mathrm{C} \text {. }\end{array}$} \\
\hline
\end{tabular}

enzyme failed to increase the efficiency of product formation (data not shown), which remained at approximately $0 \%-15 \%$ for the three NTPs (Table 3 ).

E. Coumarin-alkynylamino-UTP. Of all the substrates investigated here, coumarin-UTP was the substance that, on the basis of its incorporation, was the most readily accepted by terminal transferase. A plateau of $83 \%$ of monoaddition was reached in cacodylate/DMSO (Table 3).

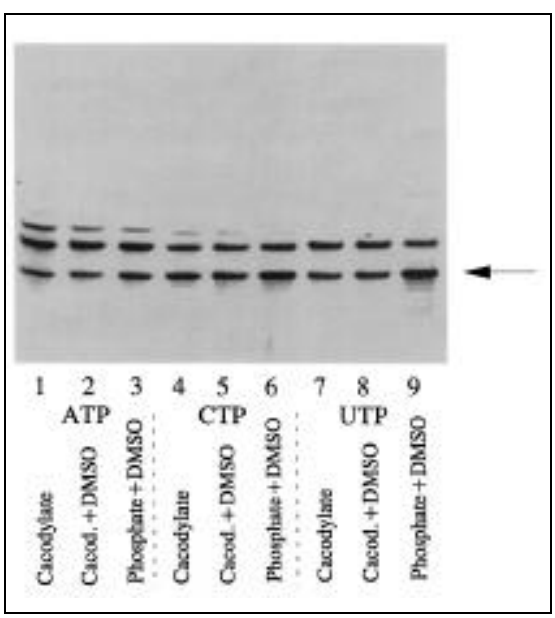

Figure 4. Terminal addition of fluorescein alkynyl-ATP, -CTP and -UTP under three different buffer conditions. The reactions were carried out as described in Materials and Methods. The buffers correspond to Nos. 1, 4 and $6+$ DMSO, respectively, in Table 2. Bands were located by staining with Stains-All. The position of the unlabeled primer is marked by an arrow.

\section{Sequence Reactions}

In the case with fluorescein-allylamino-UTP (4), labeling of DNA sequencing primers to an extent of $60 \%$ was sufficient to generate high-quality sequence data. In order to consider the 3 -terminal labeling of sequencing primers with fluorescein-ATP and -CTP as a routine procedure despite a product yield of only about $50 \%$, the quality of DNA sequence data obtained with such primers had to be examined. Figure 5 exemplifies automated sequence traces obtained with primers labeled 3'-terminally with fluoresceinCTP or fluorescein-ATP using the cycle sequencing protocol in conjunction with Pharmacia Biotech's A.L.F. DNA Sequencer. These may be compared with the sequences acquired with the same primers bearing a $5^{\prime}$-terminal fluorescein label.

Since clearly the nature of the base of the nucleotide to be added to the primer plays a role in determining the incorporation efficiency, it was, for purposes of designing sequencing primers, of interest to examine if the partner in this reaction, the 3 '-terminal base of the primer, also had an influence on the product formation. For this purpose a set of four primers was designed with the sequence $(\mathrm{T})_{16} \mathrm{~N}$, where $\mathrm{N}$ was one of the four nucleotides $\mathrm{A}, \mathrm{C}, \mathrm{G}$ or T. Labeling of these primers with fluores- 
Table 4. Effect of Potential Secondary Structures on the Incorporation Efficiency of FluoresceinUTP

\begin{tabular}{|lcc|}
\hline Primer & \multicolumn{1}{c}{$\begin{array}{c}\text { Primer Sequence and } \\
\text { Hairpin Prediction }\end{array}$} & $\begin{array}{c}\text { Incorporation } \\
\text { of Fluorescein-UTP } \\
\text { (\%) }\end{array}$ \\
\hline T3A & 5' ATTAACCCTCACTAAAAAGGGA & 78 \\
Reverse & 5' CAGGAAACAGCTATGACC & 72 \\
Forward & 5' GTAAAACGACGGCCAGTG & 60 \\
SP6 & 5' ACCTTATGTATCATACACAT & 42 \\
Standard sequencing primers terminating with A, C, G or T were labeled with fluo- \\
rescein allylamino-rUTP under standard conditions (4), and the incorporation was \\
assessed by electrophoresis, staining and densitometry. Secondary structure pre- \\
diction was performed using OLIGO 5.0 (National Biosciences, Plymouth, MN, \\
USA). Sequences predicted to give rise to hairpin structures are underlined. \\
\hline
\end{tabular}

Following the analyses with sets of ribonucleotides bearing the same fluorescent tag, it has become clear that, as in the case of biotin-dNTPs (2), the nature of the base has some effect on the efficiency and extent of the reaction, and for none of the nucleotides was the incorporation comparable to unmodified rNTPs (11). However, the label attached to the base is a critical determinant in affecting the substrate properties of the nucleotide; the Texas Red label inhibited incorporation, while fluorescein was able to stimulate multiple additions. A more detailed examination of the structures of the dyes (Figure 1) that have been used leads to the following conclusions. Coumarin-UTP was by far the best substrate, in terms of incorporation efficiency. However, as it is not structurally closely related to the other dyes considered here, it will be ignored for the purposes of this comparison. Of the dyes based on the fluorescein-type structure, fluorescein itself is the simplest, being represented as a quinoid tautomeric xanthene ring structure and a carboxylate side chain. The next in the series of structural analogues is tetramethylrhodamine, where the oxygen atoms of the quinoid system are replaced by dimethylamino residues carrying a tautomeric positive charge (pyronine system). In lissamine, these dimethylamino groups are replaced by diethylamino groups, while the carboxylate is replaced by a sulphonate. Texas Red may also be regarded being derived from fluorescein, but now the diethyl-substituents of the exocyclic amines of lissamine are replaced by dibutyl chains with cycliza- tion onto the xanthene system. The sulphonate and its associated negative charge (at neutral $\mathrm{pH}$ ), introduced in lissamine, is retained in Texas Red. As far as the substrate properties of nucleotides derivatized with these dyes are concerned, one observes for terminal transferase the following preference series for a given nucleotide: fluorescein $\gg$ tetramethylrhodamine > lissamine $=$ Texas Red. Although this series reflects the increase in steric size of the dyes, the introduction of a relatively small dimethylamino group (compared with the unaltered bulky aromatic system) on going from fluorescein to tetramethylrhodamine causes a disproportionate reduction in activity. One may, then, consider the introduction of a positive charge on the xanthene nucleus, which is conserved from tetramethylrhodamine through to lissamine as a major factor in their reduced activity. The change from a carboxylate to a sulphonate side chain should be of little consequence since at neutral $\mathrm{pH}$ both species will be substantially ionized.

Reaction conditions optimal for a particular label and independent of the attached base can be defined only to a limited extent. One observes, even in the case of fluorescein, a drop in monoaddition activity of $50 \%$ for labeled UTP when changing from cacodylate/ DMSO to phosphate/DMSO, whereas the analogous ATP and CTP are hardly affected. While not the aim of this investigation, other differences in the substrate activity of these closely related nucleotides are revealed by a more detailed examination of the products formed under the reaction conditions 


\section{Research Reports}

A
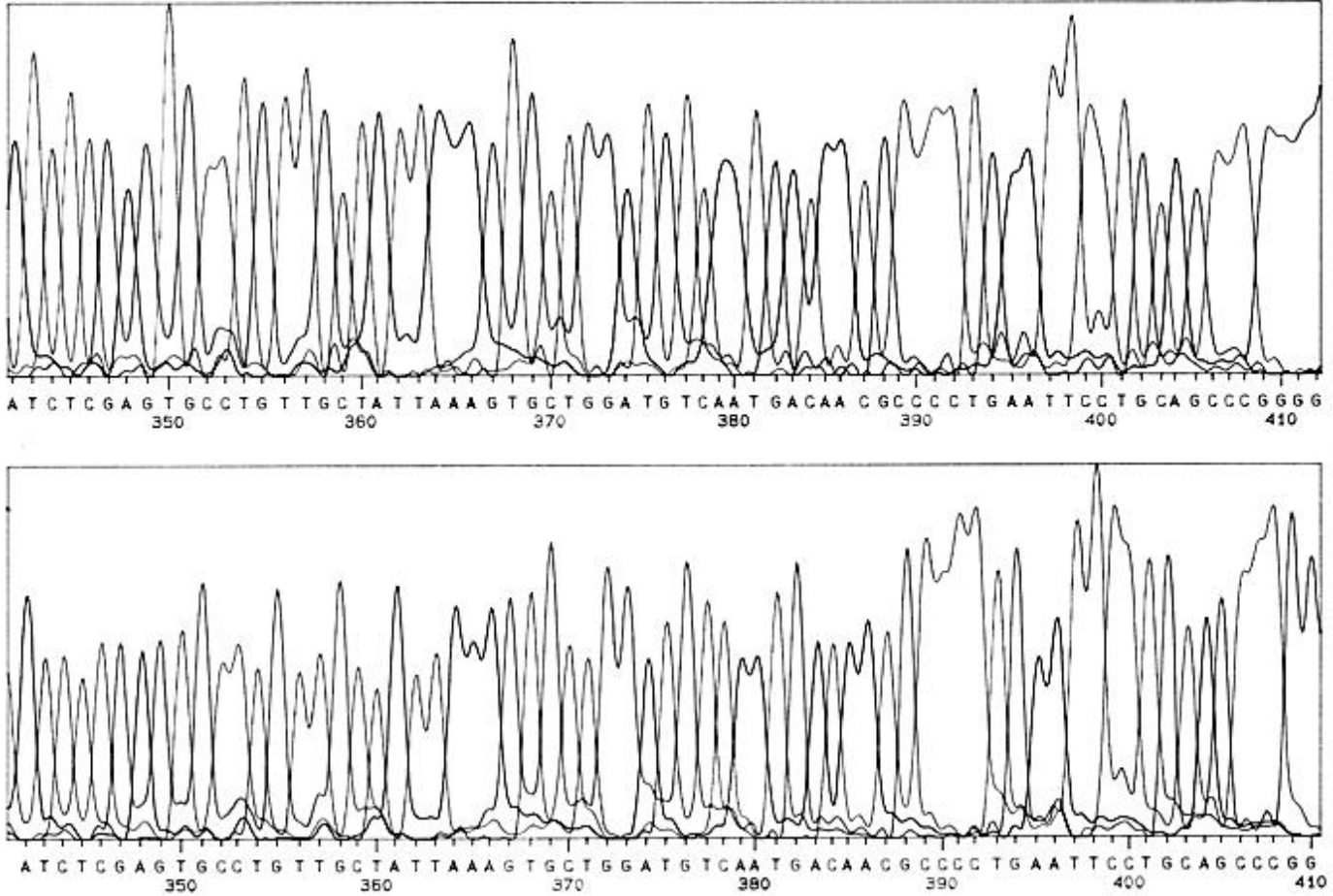

B
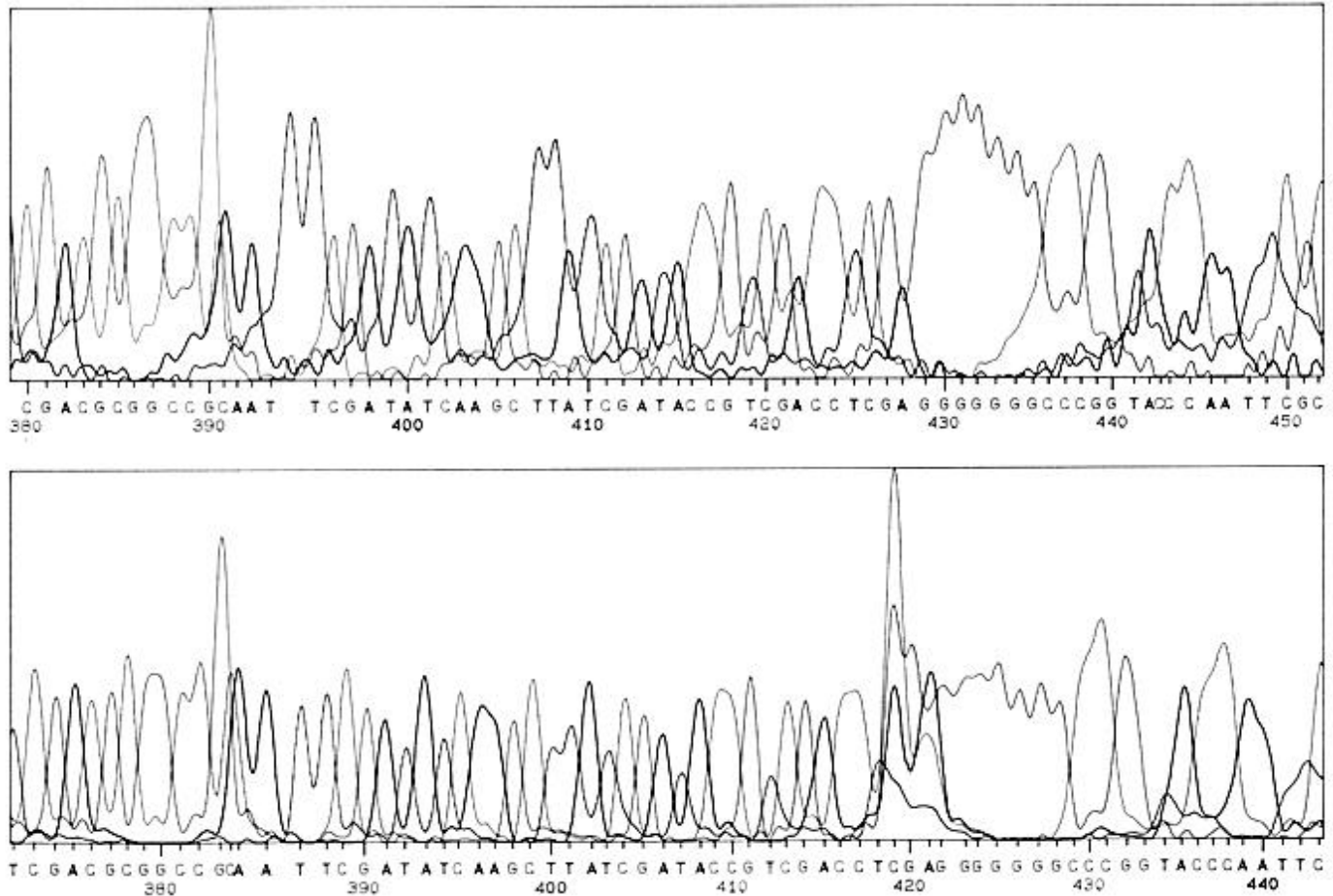

Figure 5. Sequence traces obtained with $3^{\prime}$-terminally labeled primers as compared to the identical region acquired with conventional 5 ' fluorescent primers. A section of a mammalian gene, cloned into Bluescript ${ }^{\circledR}$ (Stratagene, La Jolla, CA, USA), was sequenced, as described in Materials and Methods, using Thermo Sequenase. Panel A: Top: Bluescript KS primer was 3'-terminally labeled with fluorescein alkynylamino-ATP as described. Bottom: The same region of sequence using a fluorescent KS primer which had been synthesized by incorporation of FluorePrime at the 5' end. Panel B: Top: Bluescript SK primer was 3'-terminally labeled with fluorescein alkynylamino-CTP as described. Bottom: The same region of sequence using a fluorescent SK primer that had been synthesized by incorporation of FluorePrime at the $5^{\prime}$ end. Note that the compression at position 384 is present in both cases. 
tested. Thus, as in the case of unmodified rNTPs (11), reduced activity is seen when $\mathrm{Mg}^{++}$replaces $\mathrm{Co}^{++}$in the case of the alkynyl derivative, but product formation is virtually abolished in the presence of $\mathrm{Mn}^{++}$. For allylaminolinked UTP, $\mathrm{Mn}^{++}$is more stimulatory than $\mathrm{Mg}^{++}$. However, the different activity between alkynylamino-linked and allylamino-linked nucleotides is, on the whole, minimal.

The establishment of conditions for mononucleotide addition permits the fluorescent labeling of DNA sequencing primers (4). Such primers are of central importance in automated sequencing strategies employing fluorescence-based machines. Currently, these machines depend on lasers of different wavelengths. First-generation sequen-cers were based on either a scanning system simultaneously detecting the emission of four dyes (fluorescein, tetramethylrhodamine, Texas Red and
4', $5^{\prime}$-dichloro- $2^{\prime}, 7^{\prime}$-dimethoxyfluorescein $[\mathrm{JOE}])(\mathrm{PE} / \mathrm{ABI})$ or the fixed wavelength detection of fluorescein fluorescence (Pharmacia Biotech). Recently, the use of red or infra-red lasers has gained popularity (e.g., ALFexpress $^{\mathrm{TM}}$ from Pharmacia Biotech, Vistra from Amersham and another instrument from Li-Cor (Lincoln, NE, USA). In view of the limited commercial availability of ribonucleotides labeled with the appropriate fluorescent dye, attention has been focused here on those compounds that could be of immediate use in DNA sequencing. Of the dyes tested, Texas Red is the only one that could have applications in two commercial systems (ALFexpress and Vistra). Unfortunately, it is one dye in this study that, when attached to a base, drastically reduces its incorporation by terminal transferase. At present, in order to use primers labeled in this way, separation from unlabeled material is required. Such purification, while feasible (e.g., by gel electrophoresis or other techniques), detracts from the convenience of the labeling procedure. This factor, together with the current unavailability of JOE-rNTPs, means that this method is of value to users of $\mathrm{PE} / \mathrm{ABI}$ 's sequencers only as far as fragment analysis is concerned, using fluorescein.

In the case of the fluorescein nucleotides, which are of interest to users of Pharmacia Biotech's A.L.F. system, the efficient conversion of a primer to its fluorescent derivative, first described for fluorescein-allylamino-UTP (4), is maintained in the alkynylamino series by an appropriate buffer change. These primers can be and have been used for DNA sequencing directly or following a simple spin column separation of the unincorporated nucleotide either isothermally with T7 DNA polymerase or by thermal cycling using 
$\Delta T a q^{\circledR}$ (4), SequiTherm (13) or Thermo Sequenase polymerases. The quality of the sequence data, in terms of the length of the read, is indistinguishable from that obtained with $5^{\prime}$-terminally labeled primers. Fluorescein-UTP has been used routinely in the design and synthesis of walking primers for a number of years (4); fluorescein-CTP and -ATP have played a significant role in the ability to use pre-synthesized primers for the completion of a genome project covering $140 \mathrm{~kb}(7)$.

The avoidance of secondary structure should, in any event, be considered during primer design and is also of relevance for the labeling reaction. Sequences permitting the formation of 3 'terminal hairpin structures including the terminal base itself may be poorer substrates for terminal transferase; the identity of this 3 '-terminal base does not seem to be a determinant in labeling efficiency. With primers that include secondary structure motifs, labeling might be expected to be at a level no longer acceptable for use in DNA sequencing. However, as shown previously (4), a labeled fraction comprising $50 \%$ of the primer is sufficient to give satisfactory sequence data. Routine use of this procedure has confirmed that all primers obeying standard primer design requirements may be successfully labeled and applied to DNA sequencing. The current unavailability of fluorescein-GTP, while unfortunate, is not of crucial importance in the de novo design of walking primers; however, it does restrict the use of previously synthesized primers to some extent.

Chemically labeled primers are uneconomical for large-scale sequencing projects but, when used, provide more data per run than the alternative dyeterminator approach (8). The enzymatic method introduced previously and extended here provides a convenient, rapid and financially attractive alternative pathway to obtaining dye-labeled primers that have been shown to perform quantitatively and qualitatively identically to conventional chemically synthesized dye-primers.

\section{ACKNOWLEDGMENTS}

I am grateful to E. Schiefermayr for excellent technical support. I am in- debted to Dr. T. Knott, Amersham, UK, for examining the potential of the Texas Red-labeled primers in the Vistra system. This work was funded, in part, by the Deutsche Forschungsgemeinschaft (SFB 388).

\section{DEDICATION}

This publication is dedicated to the memory of Professor Hans Kössel.

\section{REFERENCES}

1.Feix, G. 1971. Oligoribonucleotides as primers for terminal deoxynucleotidyl transferase. FEBS Lett. 18:280-282.

2.Flickinger, J.L., G. Gebeyehu, G. Buchman, A. Haces and A. Rashtchian. 1992. Differential incorporation of biotinylated nucleotides by terminal deoxynucleotidyl transferase. $\mathrm{Nu}-$ cleic Acids Res. 20:2382.

3.Igloi, G.L. 1996. Enzymatic fluorescence and biotin labeling of primers for PCR sequencing. Methods Mol. Biol. 65:23-28.

4.Igloi, G.L. and E. Schiefermayr. 1993. Enzymatic addition of fluorescein- or biotin-riboUTP to oligonucleotides results in primers suitable for DNA sequencing and PCR. BioTechniques 15:486-497.

5.Kössel, H. and R. Roychoudhury. 1971. Synthetic polynucleotides. The terminal addition of riboadenylic acid to deoxyoligonucleotides by terminal deoxynucleotidyl transferase as a tool for the specific labeling of deoxyoligonucleotides at the $3^{\prime}$ ends. Eur. J. Biochem. 22:271-276.

6.Kumar, A., P. Tchen, F. Roullet and J. Cihen. 1988. Nonradioactive labeling of synthetic oligonucleotide probes with terminal doexynucleotidyl transferase. Anal. Biochem. 169:376-382.

7.Maier, R.M., K. Neckermann, G.L. Igloi and H. Kössel. 1995. Complete sequence of the maize chloroplast genome: gene content, hotspots of divergence and fine tuning of genetic information by transcript editing. J. Mol. Biol. 251:614-628.

8.Naeve, C.W., G.A. Buck, R.L. Niece, R.T. Pon, M. Robertson and A.J. Smith. 1995. Accuracy of automated DNA sequencing: a multi-laboratory comparison of sequencing results. BioTechniques 19:448-453.

9.Ratliff, R.L. 1981. Terminal deoxynucleotidyl transferase, p. 105-118. In P.D. Boyer (Ed.), The Enzymes. Vol. 14a. Academic Press, New York.

10.Rosemeyer, V., A. Laubrock and R. Seibl. 1995. Nonradioactive 3 '-endlabeling of RNA molecules of different lengths by terminal deoxynucleotidyl transferase. Anal. Biochem. 224:446-449.

11.Roychoudhury, R., E. Jay and R. Wu. 1976. Terminal labeling and addition of homopolymer tracts to duplex DNA fragments by terminal transferase. Nucleic Acids Res. 3:863-878.

12.Roychoudhury, R. and H. Kössel. 1971. Synthetic oligonucleotides. Enzymatic syn- thesis of ribonucleotide terminated oligodeoxynucleotides and their use as primers for the enzymatic synthesis of polydeoxynucleotides. Eur. J. Biochem. 22:310-320.

13.Schiefermayr, E. and G.L. Igloi. 1995. Degradation of DNA sequencing primers by a terminal transferase-associated exonuclease. Anal. Biochem. 230:180-182.

14.Tu, C.-P.D. and R. Wu. 1980. Sequence analysis of short DNA fragments. Methods Enzymol. 65:620-638.

Received 16 January 1996; accepted 21 May 1996.

\author{
Address correspondence to: \\ Gabor L. Igloi \\ Institut für Biologie III \\ Universität Freiburg \\ Schänzlestr. 1 \\ D-79104 Freiburg, Germany \\ Internet: igloi@oligo.biologie.uni-freiburg.de
}

